\title{
Quantum spin Hall effect induced by nonmagnetic and magnetic staggered potentials
}

\author{
Huaiming Guo, ${ }^{1, *}$ Shiping Feng, ${ }^{2}$ and Shun-Qing Shen ${ }^{3}$ \\ ${ }^{1}$ Department of Physics and Center of Theoretical Physics, Capital Normal University, Beijing 100048, China \\ ${ }^{2}$ Department of Physics, Beijing Normal University, Beijing 100875, China \\ ${ }^{3}$ Department of Physics and Center of Computational and Theoretical Physics, The University of Hong Kong, Pokfulam Road, Hong Kong
}

(Received 13 October 2010; published 31 January 2011)

\begin{abstract}
We conducted a comparative study of the quantum spin Hall (QSH) effects induced by nonmagnetic and magnetic staggered potentials, respectively, and show that they have the same effect in driving the topological phase transition. The result implies that both time-reversal $(\mathcal{T})$ preserving and breaking systems can host a QSH effect. We also investigate the stability of the resulting QSH effect for disorder and find that, for $\mathcal{T}$ invariant systems, the edge states are always robust while those of the $\mathcal{T}$ breaking system are also robust if there is additional symmetry in the system.
\end{abstract}

DOI: 10.1103/PhysRevB.83.045114

PACS number(s): 73.43.-f, 72.25.Hg, 73.20.-r, 85.75.-d

\section{INTRODUCTION}

Recently, the field of the topological insulator (TI) has attracted a great deal of interest, due to its exotic physical properties as well as potential applications, such as spintronics, quantum computing, etc. ${ }^{1-3}$ Many materials have been predicted and discovered to show TI phases [including $\mathrm{HgTe} / \mathrm{CdTe}$ quantum wells $(\mathrm{QWs}){ }^{4,5}$ bismuth antimony alloys ${ }^{6,7} \mathrm{Bi}_{2} \mathrm{Se}_{3}$, $\mathrm{Bi}_{2} \mathrm{Te}_{3}$, and $\mathrm{Sb}_{2} \mathrm{Te}_{3},{ }^{8-11}$ Heusler compounds, ${ }^{12,13}$ Tl-based ternary chalcogenide series, ${ }^{14,15}$ etc.]. The findings of the real materials not only provide a platform to test the predictions of many unusual phenomena exhibited by $\mathrm{TI},{ }^{16-19}$ but also inspire more theoretical studies on TI.

The study of TI begins with the theoretical proposal of quantum spin Hall (QSH) effect in graphene by Kane and Mele. ${ }^{20,21}$ They expected that spin-orbit coupling will convert graphene from an ideal two-dimensional (2D) semimetallic state to a QSH insulator. The resulting QSH insulator is topologically distinct from a band insulator, so it is referred to as TI. However, the calculations have suggested that the spin-orbit coupling in graphene is too small to reveal the QSH effect experimentally. ${ }^{22}$ Remarkably, in 2007, the QSH effect was realized in $\mathrm{HgTe} / \mathrm{CdTe}$ QWs following the theoretical suggestion of Bernevig, Hughes, and Zhang. ${ }^{4,5}$ Later, although many studies have been carried out for identifying new physical systems that will possess topological nontrivial phases, until now, the 2D TI was only found experimentally in $\mathrm{HgTe} / \mathrm{CdTe} \mathrm{QWs}$. In the low-energy effective theory, the QSH effect can be understood from the Dirac Hamiltonian with masses. ${ }^{23,24}$ The relative signs of the masses at the Dirac points determine the phases of the system. Alternately, it can also be understood from band inversion, which is the mechanism of the TI phase in $\mathrm{HgTe} / \mathrm{CdTe}$ QWs. The two methods are equivalent since the occurrence of band inversion corresponds to changing the sign of one Dirac mass and causes a topological phase transition, which cannot happen without closing the gap.

Spin-orbit coupling is a necessary condition for the existence of TI. Its role is to induce a gap in the Dirac dispersion and to ensure that the gap is finite everywhere in the Brillouin zone (BZ). It also has been known that nonmagnetic and magnetic staggered potentials can perturb the Dirac dispersion and can induce a gap. Such terms can be obtained by the proximity effect to the corresponding orders [charge density wave $(\mathrm{CDW})$ and antiferromagnetism $(\mathrm{AF})] .{ }^{25}$ When these terms coexist, their interplay will determine the phase of the system. In this paper, we study the interplay of these terms. We start from a trivial insulator with spin-orbit coupling and introduce nonmagnetic and magnetic staggered potentials with checkerboard and stripe patterns into the system. We find that, when the strength of the potential is strong enough, a band inversion occurs, and the system shows QSH effect. Especially, the QSH effect induced by magnetic staggered potential breaks $\mathcal{T}$ symmetry, in contrast to the previously studied QSH effect.

\section{THE MODEL FOR HgTe/CdTe QWs}

To be concrete, we study a model describing $\mathrm{HgTe} / \mathrm{CdTe}$ QWs. It resides on a square lattice with four orbit states $|s, \uparrow\rangle$, $\left|p_{x}+i p_{y}, \uparrow\right\rangle,|s, \downarrow\rangle$, and $\left|\left(p_{x}-i p_{y}\right), \downarrow\right\rangle(\uparrow, \downarrow$ denote the electron's spin) on each site. In the momentum space, the Hamiltonian is written as

$$
\begin{aligned}
H_{0}(\mathbf{k})= & {\left[4 D-2 D\left(\cos k_{x}+\cos k_{y}\right)\right] I } \\
& +\left[M+4 B-2 B\left(\cos k_{x}+\cos k_{y}\right)\right] \sigma_{z} \\
& +2 A \sin k_{x} s_{z} \otimes \sigma_{x}+2 A \sin k_{y} \sigma_{y} .
\end{aligned}
$$

Here, $\vec{\sigma}$ and $\vec{s}$ are Pauli matrices representing the orbit and the electron's spin, and $I$ is the identity matrix. $A, B, D$, and $M$ are four independent parameters. The tight-binding Hamiltonian can be directly obtained by a lattice regulation of the effective low-energy Hamiltonian describing the physics of $\mathrm{HgTe} / \mathrm{CdTe}$ QWs. We can also view it as a simple toy model conveniently describing both topological and ordinary phases of noninteracting electrons in $2 \mathrm{D}$. The energy spectrum of $H_{0}(\mathbf{k})$ has two double degenerate branches $E_{\mathbf{k}}=$ $\left(4 D-D_{\mathbf{k}}\right) \pm \sqrt{\left(2 A \sin k_{x}\right)^{2}+\left(2 A \sin k_{y}\right)^{2}+\left(\tilde{M}-B_{k}\right)^{2}}$, where $\quad \tilde{M}=(M+4 B), \quad B_{\mathbf{k}}=2 B\left(\cos k_{x}+\cos k_{y}\right) \quad$ and $D_{\mathbf{k}}=2 D\left(\cos k_{x}+\cos k_{y}\right)$. At half-filling, depending on the values of $M$ and $B$, the system can be QSH or a trivial insulator.

The system defined by Eq. (1) is invariant under $\mathcal{T}$ and spatial inversion. Since the inversion operator $\mathcal{P}$ operating on $p$-type orbit generates a minus sign, the inversion operator writes $\mathcal{P}=I \otimes \sigma_{z}$. Thus, the signs of $\tilde{M}-B_{\mathbf{k}}$ at the four timereversal invariant momenta (TRIM) determine the phase of the system, i.e., $M(M+4 B)^{2}(M+8 B)<0(>0)$ for the QSH (trivial) insulator. ${ }^{26,27}$ In this paper, we restrict our calculations in the parameter range describing $\mathrm{HgTe} / \mathrm{CdTe} \mathrm{QWs}$, where 

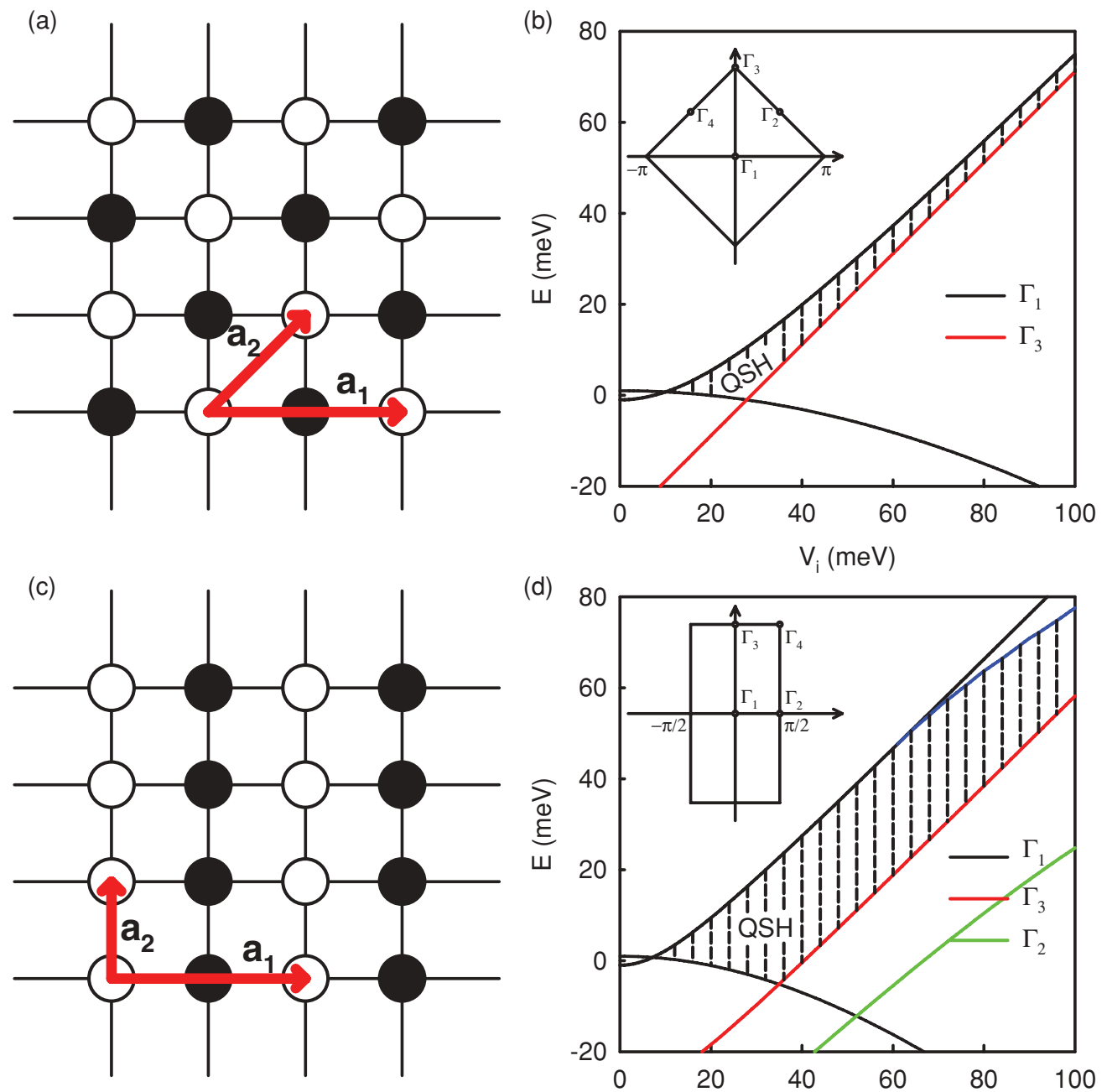

FIG. 1. (Color online) (a) The staggered potential with checkerboard pattern, (c) stripe pattern, and [(b) and (d)] the band evolvements at TRIM with the potential strength corresponding to the patterns in (a) and (c), respectively. The open and filled circles on lattice sites in (a) and (c) represent on-site potentials with equal values but opposite signs. The new lattice vectors and new BZs are shown in (a) and (c) and the insets of (b) and (d). The blue (top) curve in (d) is a band evolving at other momenta that will determine the gap size at large potential strength. The range with vertical dashed lines in (b) and (d) marks the gap of the induced topological phase. The parameters are fixed for all calculations in this paper to be $A=36.45 \mathrm{meV}, B=27.44 \mathrm{meV}, D=20.48 \mathrm{meV}$, and $M=1 \mathrm{meV}$.

$B>|M|>0$. Since the TRIM $\Gamma_{1}(\mathbf{k}=[0,0]$ in the BZ, see the inset of (b) in Fig. 1) dominates the physics in the range, the Hamiltonian describes the QSH effect for $M<0$ and a trivial insulator for $M>0$. Experimentally, the gap parameter $M$ can be continuously tuned from a positive value for thin QWs with thickness $d<d_{c}$ to a negative value for thick QWs with $d>d_{c}$ $\left(d_{c}\right.$ is a critical thickness and equals $6.3 \mathrm{~nm}$ for $\mathrm{HgTe} / \mathrm{CdTe}$ QWs). ${ }^{4,5}$ The gap parameter $M$ in Eq. (1) represents an on-site potential, which has a different sign for $s$-type and $p$-type orbits. When changing its sign, one of the occupied bands changes from $p$ type $(M>0)$ to $s$ type $(M<0)$. Since the two kind of orbits have different parities, a band inversion will induce a topological phase transition.

\section{NONMAGNETIC AND $s_{z}$-CHANNEL MAGNETIC STAGGERED POTENTIALS}

To drive the system into QSH phase, a band inversion is needed. So it is interesting to seek ways other than tuning the gap parameter $M$ to generate the band inversion. Later, we fix $M>0$ when the system is a trivial insulator and find ways to inverse the bands at TRIM $\Gamma_{1}$. A natural thought is to enlarge the Hamiltonian, which makes itv possible to add more terms to it. First, we consider putting the system on a checkerboard square lattice (CSL), which can introduce alternating potential with a checkerboard pattern. In this case, the unit cell is doubled. The Hamiltonian $H_{0}(\mathbf{k})$ is enlarged to $8 \times 8$ and becomes

$$
\begin{aligned}
H_{1}(\mathbf{k})= & 4 D I-2 D\left(\cos k_{x}+\cos k_{y}\right) \tau_{x} \\
& +(M+4 B) \sigma_{z}-2 B\left(\cos k_{x}+\cos k_{y}\right) \tau_{x} \otimes \sigma_{z} \\
& +2 A \sin k_{x} \tau_{x} \otimes s_{z} \otimes \sigma_{x}+2 A \sin k_{y} \tau_{x} \otimes \sigma_{y} .
\end{aligned}
$$

Here, $\vec{\tau}$ is the Pauli matrix describing the two sublattices. We have been able to identify two interesting on-site terms: (i) a nonmagnetic staggered potential (or CDW potential) $V_{1} \tau_{z}$; (ii) a magnetic staggered potential (or AF potential) $V_{2} \tau_{z} \otimes$ $s_{z}$. (The magnetization also can lie in the plane and couples 
TABLE I. The eigenvectors for the Hamiltonian at TRIM $\Gamma_{1}$ and their parities. Here, $\phi_{1 \pm}=\frac{\tilde{B}_{ \pm}+V_{i}}{4 B \pm 4 D}$ and $\phi_{2 \pm}=\frac{\tilde{B}_{ \pm}-V_{i}}{4 B \pm 4 D}$. The superscripts for the values of the eigenvectors represent the corresponding position in the eigenvectors.

\begin{tabular}{lllr}
\hline \hline No. & Eigenvector $(\mathrm{CDW})$ & \multicolumn{1}{c}{ Eigenvector $(\mathrm{AF})$} & Parity \\
\hline 1 & $\left(-\phi_{2-}^{2}, 1^{6}\right) ;\left(-\phi_{2-}^{4}, 1^{8}\right)$ & $\left(-\phi_{2-}^{2}, 1^{6}\right) ;\left(-\phi_{1-}^{4}, 1^{8}\right)$ & -1 \\
2 & $\left(\phi_{1-}^{2}, 1^{6}\right) ;\left(\phi_{1-}^{4}, 1^{8}\right)$ & $\left(\phi_{1-}^{2}, 1^{6}\right) ;\left(\phi_{2-}^{4}, 1^{8}\right)$ & -1 \\
3 & $\left(\phi_{2+}^{3}, 1^{7}\right) ;\left(\phi_{2+}^{1}, 1^{5}\right)$ & $\left(\phi_{1+}^{3}, 1^{7}\right) ;\left(\phi_{2+}^{1}, 1^{5}\right)$ & 1 \\
4 & $\left(-\phi_{1+}^{3}, 1^{7}\right) ;\left(-\phi_{1+}^{1}, 1^{5}\right)$ & $\left(-\phi_{2+}^{3}, 1^{7}\right) ;\left(-\phi_{1+}^{1}, 1^{5}\right)$ & 1 \\
\hline \hline
\end{tabular}

with the in-plane components of the electron's spin, generating terms, such as $\tau_{z} \otimes s_{x}$ or $\tau_{z} \otimes s_{y}$. We will discuss these terms later.) The former term preserves $\mathcal{T}$ symmetry while the latter breaks. Including the preceding terms for Eq. (2), although we cannot obtain the analytic forms of the energy spectrums, they are the same for the two different cases. The system remains gapped, and the occupied bands evolve with the strength of $V_{i}(i=1,2)$.

At TRIM $\Gamma_{1}$, the energy eigenvalues are: (1) $4 D-\tilde{M}-$ $\tilde{B}_{-}$; (2) $4 D-\tilde{M}+\tilde{B}_{-}$; (3) $4 D+\tilde{M}-\tilde{B}_{+}$; (4) $4 D+\tilde{M}+$ $\tilde{B}_{+}$, where $\tilde{B}_{ \pm}=\sqrt{(4 D \pm 4 B)^{2}+V_{i}^{2}}$ and each is double degenerate. The corresponding eigenvectors and their parities can also be obtained, which are listed in Table I. The band evolvement with the strength $V_{i}$ is shown in (b) of Fig. 1. At half-filling and for $V_{i}=0$, bands (1) and (2) are occupied. Since we choose $M>0$, the system is a trivial insulator. As $V_{i}$ increases, bands (2) and (3) first approach each other, and at a critical value of $V_{i}$, the filling for the two bands will interchange. Bands (2) and (3) consist of electrons in the $p$-type and $s$-type orbits, respectively, and have opposite parities. For the case with the CDW term, the band inversion will induce a topological phase transition and will drive the system into the QSH phase. It is interesting that the AF term has the same effect as the CDW term. As we further increase the strength of the potential, the system remains in QSH phase until another band inversion occurs at another TRIM, which happens at a potential strength greater than $100 \mathrm{meV}$. The gap range of the resulting QSH insulator is also denoted in (b) of Fig. 1. At small potential strength, it is determined by bands (2) and (3) and increases with increasing potential strength. Then, a band evolving at TRIM $\Gamma_{3}(\mathbf{k}=[0, \pi]$ in the BZ, see the inset of (b) in Fig. 1) becomes the lower restriction of the gap and restricts its further increase. It is interesting to note that the behavior is similar to what happens in the topological Anderson insulator, where the phase diagram has to be obtained by conductivity calculations since the system has no translation symmetry in the presence of disorder. ${ }^{28-31}$

We can also put the system on a stripe square lattice (SSL), as shown in (c) of Fig. 1. The Hamiltonian $H_{0}(\mathbf{k})$ becomes

$$
\begin{aligned}
H_{2}(\mathbf{k})= & \left(4 D-2 D \cos k_{y}\right) I-2 D \cos k_{x} \tau_{x} \\
& +\left(M+4 B-2 B \cos k_{y}\right) \sigma_{z}-2 B \cos k_{x} \tau_{x} \otimes \sigma_{z} \\
& +2 A \sin k_{x} \tau_{x} \otimes s_{z} \otimes \sigma_{x}+2 A \sin k_{y} \sigma_{y} .
\end{aligned}
$$

Similarly, a stripe CDW $\left(\tau_{z}\right)$ or AF term $\left(\tau_{z} \otimes s_{z}\right)$, which has the same form as its counterpart on CSL, can be added to the previous Hamiltonian. The energy spectrums for both cases are still the same. The band evolvements with the potential strength at TRIM are shown in (d) of Fig. 1. The CDW or AF term on SSL can also induce a band inversion and can drive the system into QSH phase. However, compared to the case in the CSL, here, the band inversion occurs at a smaller potential strength and the resulting QSH insulator has a bigger gap.

So, adding the CDW or AF term to the system can induce a band inversion. Although they have different properties under $\mathcal{T}$ transformation, they have the same effects on band inversion. The reason is that the combined Hamiltonian at TRIM $\Gamma_{1}$ can be decoupled for each orbit and spin. Each decoupled Hamiltonian has a $2 \times 2$ form and has two subbands. The CDW or AF term pushes one sub-band up and the other down, which does not depend on the sign of the potential strength. Since the difference between CDW and AF terms is that the AF term acts on a different spin with a different sign, they have the same effect on changing the band structure.

The CDW term preserves $\mathcal{T}$ symmetry, and such systems can be described by the $Z_{2}$ topological invariant. ${ }^{21,32}$ Since our system has inversion symmetry, the $Z_{2}$ invariant can be determined from the knowledge of the parities of the occupied band eigenstates at the four TRIM. When the band inversion occurs as we increase the potential strength, the value of the $Z_{2}$ invariant also changes its sign and becomes nontrivial, indicating that the system is in QSH phase. For the case with the AF term, the $\mathcal{T}$ symmetry is broken, and the $Z_{2}$ topological invariant is inapplicable. However, the spin Hall conductance of the resulting QSH phase still shows the quantized value $\frac{e}{2 \pi} \cdot{ }^{33}$ So the underlying topological invariant is a spin Chern number, which describes the quantized spin Hall conductivity. The concept of the spin Chern number has appeared in the
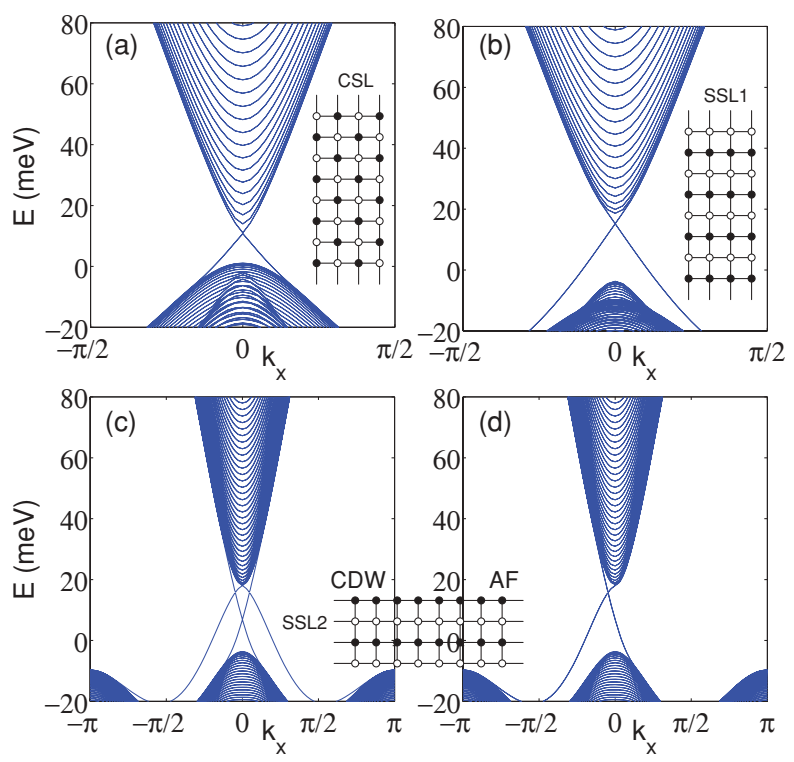

FIG. 2. (Color online) One-dimensional energy bands with a strip geometry (shown in the inset) for (a) a checkerboard pattern and (b), (c), and (d) a stripe pattern. In (a) and (b), the energy bands are the same for the cases with the CDW and AF terms. (c) and (d) have different edges with (b), and the energy bands with the CDW term (c) are different from those with the AF term (d). A strip of width $N_{y}=60$ unit cell with open boundary conditions along $y$ and infinite along $x$ is used with the staggered potential strength $30 \mathrm{meV}$, when the system is in the band-inverted phase. 
recent literature, which has its definition for systems with spin $s_{z}$ conservation. ${ }^{34}$ For the $\mathcal{T}$ invariant systems, it is equivalent to the $Z_{2}$ topological invariant. ${ }^{32}$

To further support our identification of the topological phase, we have performed numerical diagonalization of Hamiltonians (2) and (3) with the CDW or AF term using a strip geometry in the range of parameters where the system is in band-inverted phase. In accord with the foregoing arguments, we find a pair of spin-filtered gapless states associated with each edge traversing the gap, which is shown in Fig. 2. The spin-filtered edge states determine the transport of charge and spin in the gap range. For a two-terminal device, the conductance is contributed by two conducting channels on the edges and gets the quantized value $2 e^{2} / h$. For a fourterminal device with proper voltage on each terminal, a spin current can be generated. ${ }^{20}$

Until now, we demonstrated the existence of the QSH effect induced by the nonmagnetic and magnetic staggered potentials in a trivial insulator with a spin-orbit coupling. In the following, we study the stability of the resulting QSH effect for nonmagnetic disorder. The Hamiltonian we are considering can be decoupled for spin-up and spin-down electrons, and each describes quantum anomalous Hall (QAH) effect. The resulting QSH effect can be understood as two copies of the QAH effects. For the CDW case, the two copies for spin-up and spin-down electrons are related by $\mathcal{T}$, and the QSH effect is immune to nonmagnetic disorder. However, for the AF case, although $\mathcal{T}$ symmetry is broken, the system preserves the combined symmetry of $\mathcal{T}$ and a primitive lattice translation, which has been studied in three dimensions, and the AF topological insulator is predicted. ${ }^{35}$ So, the two copies for spin-up and spin-down electrons in the presence of the AF term are related by the combined transformation. If there is nonmagnetic disorder in the system, the combined symmetry will be broken, and the two copies will behave separately. But we still expect the combined system will be robust for disorder because each copy is in the QAH phase and robust for disorder.

To support the earlier statement, we employ the recursive Green's function method to evaluate the conductance $G$ of two-terminal devices (see the insets of Fig. 2) using Landauer-Büttiker formalism. Figure 3 shows the results of such calculations in the space of parameters $\left(E_{F}, U_{0}\right)$, where $U_{0}$ is disorder strength and the disorder is described by a random on-site potential uniformly distributed in the range $\left(-U_{0} / 2, U_{0} / 2\right)$. We only consider a single disorder realization at each point of the $\left(E_{F}, U_{0}\right)$ phase diagram. Nevertheless, this turns out to be sufficient for studying the stability of the edge states. The reason is that, if the edge states are robust for disorder, there should be a region showing quantized conductance $G=2 e^{2} / h$ in the phase diagram, and conductance $G$ in the region shows no observable fluctuations but fluctuates significantly elsewhere. The plots in Fig. 3 display conductance $G$ in a fashion that is designed to amplify the effect of fluctuations. In (a), (b), (d), and (e) of Fig. 3, regions showing no observable fluctuations exist in the phase
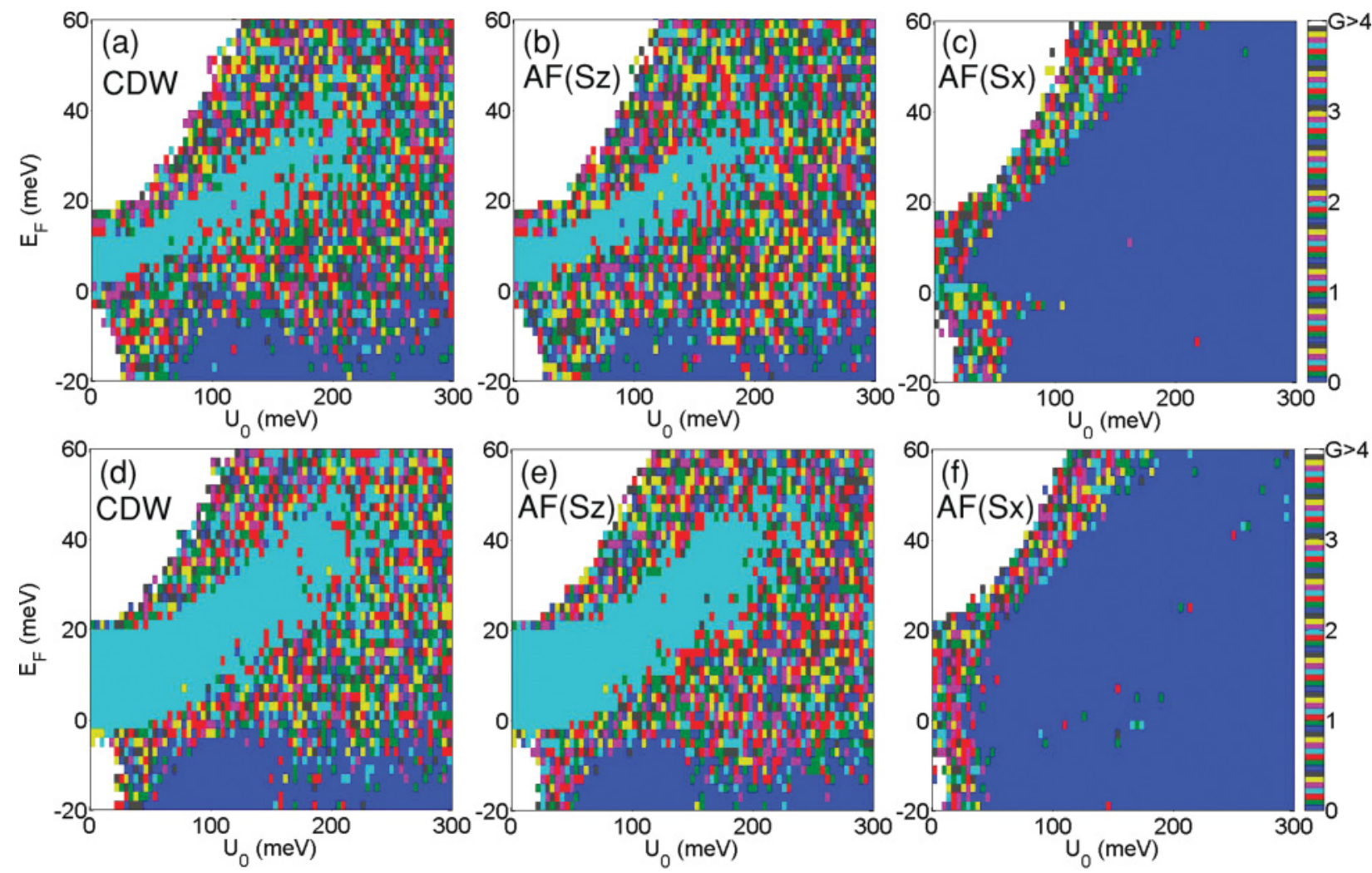

FIG. 3. (Color online) Conductance $G$ as a function of disorder strength $U_{0}$ and the Fermi level $E_{F}$. Each data point corresponds to a single disorder realization. (a)-(c) are the results with the CDW term, the AF terms in the $s_{z}$ and $s_{x}$ channels on the CSL strip respectively; (d)-(f) are corresponding results on the SSL1 strip. Here, the parameters are the same as those in Fig. 2, when the system shows the band-inverted phase in its clean form. 


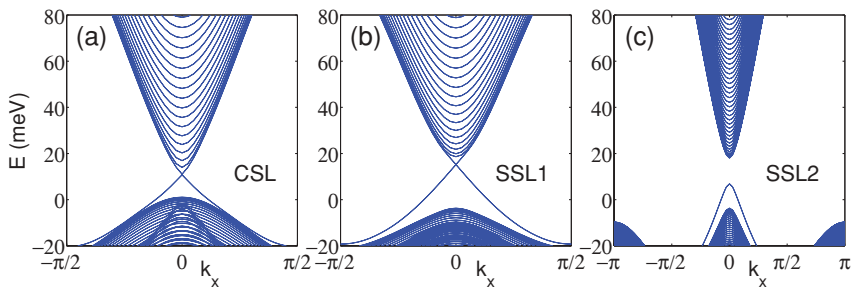

FIG. 4. (Color online) One-dimensional energy bands with the AF term in the $s_{x}$ channel on a strip geometry (a) CSL, (b) SSL1, (c) SSL2. The parameters used here are the same as those in Fig. 2.

diagrams, implying that the QSH phase induced by CDW or $\mathrm{AF}$ (in the $s_{z}$ channel) term is robust for disorder. We also carried out calculations on strip SSL2, and the results are similar to those on strip SSL1. These results are consistent with what we expect.

\section{IV. $s_{x}$-CHANNEL MAGNETIC STAGGERED POTENTIAL}

As mentioned, for the AF term, the magnetization can also lie in the plane (generating the term in the $s_{x}$ or $s_{y}$ channel), and in the following, we will focus on such terms. Including such terms for the Hamiltonian described by Eq. (2) or Eq. (3), the band evolvement at TRIM $\Gamma_{1}$ is exactly the same as that due to the $\mathrm{CDW}$ or AF potential in the $s_{z}$ channel. However, unlike the cases with the $s_{z}$ channel term, the combined Hamiltonian cannot be decoupled for spin-up and spin-down electrons (i.e., no longer is there $s_{z}$ conservation in the system). We perform numerical diagonalization of Hamiltonians (2) and (3) with the AF term in the $s_{x}$ channel (it is the same with the $s_{y}$ channel term), and the one-dimensional energy bands with a strip geometry are shown in Fig. 4. For strips CSL and SSL1 (see insets of Fig. 2), the energy spectra are similar to those in Fig. 2, and there are edge states traversing the gap in the band-inverted phase. But for strip SSL2, the edge states vanish although the system is in the band-inverted phase. The difference can be understood from the symmetries existing in the system. The AF term in the $s_{x}$ channel couples spin-up and spin-down electrons, and the system only preserves the combined symmetry of $\mathcal{T}$ and a primitive lattice translation. The existence of edge states is due to the combined symmetry.
Since the edges of strip SSL2 are ferromagnetic and break the combined symmetry, the edge states are gapped. However, because the combined symmetry on the edges of strips CSL and SSL1 is still preserved, the edge states exist. But these edge states are no longer robust for disorder, which can be shown from conductivity calculations [(c) and (f) of Fig. 3]. Here, it is disorder that breaks the combined symmetry, and the QSH phase in the system will no longer be protected. Whereas, for the system with the $s_{z}$ channel term, although ferromagnetic edges or disorder breaks the combined symmetry, the spin $s_{z}$ conservation follows to assure the existence and robustness of the edge states.

\section{CONCLUSIONS}

In conclusion, we introduce nonmagnetic and magnetic staggered potentials to a trivial insulator with spin-orbit coupling and find that they can induce a topological phase transition and drive the system into topological phase. For the nonmagnetic staggered potential, the resulting QSH phase is protected by $\mathcal{T}$ symmetry and supports the edge states on any edge, which is robust for disorder. Whereas, for the magnetic staggered potential, there is a combined symmetry of $\mathcal{T}$ and a primitive lattice translation in the system. Also, if there is an additional symmetry, i.e., spin $s_{z}$ conservation, edge states will exist on any edge in the band-inverted phase and are robust for disorder. However, in the absence of such symmetry, although edge states will exist on specific edges in the band-inverted phase, they are no longer robust for disorder. Our results imply that, although the QSH effect generally is protected by $\mathcal{T}$ symmetry, if there are additional symmetries, the QSH effect also can be found in $\mathcal{T}$ breaking systems.

\section{ACKNOWLEDGMENTS}

The authors are indebted to M. Franz, S.-P. Kou, G. Refael, Q.-F. Sun, and J.-W. Ye for stimulating discussions. Support for this work came from the funds from the Beijing Education Commission under Grant No. KM200910028008, the Ministry of Science and Technology of China under Grants No. 2011CBA00102 and No. 2011CB921700, NSFC under Grants No. 10774015 and No. 11074023, and the Research Grant Council of Hong Kong under Grants No. HKU 7051/10P and No. HKUST3/CRF/09. *hmguo@phas.ubc.ca

${ }^{1}$ J. E. Moore, Nature (London) 464, 194 (2010).

${ }^{2}$ M. Z. Hasan and C. L. Kane, Rev. Mod. Phys. 82, 3045 (2010).

${ }^{3}$ Xiao-Liang Qi and Shou-Cheng Zhang, e-print arXiv:1008.2026 (to be published).

${ }^{4}$ B. A. Bernevig, T. L. Hughes, and S.-C. Zhang, Science 314, 1757 (2006).

${ }^{5}$ M. König, S. Wiedmann, C. Brüne, A. Roth, H. Buhmann, L. W. Molenkamp, X.-L. Qi, and S.-C. Zhang, Science 318, 766 (2007).

${ }^{6}$ D. Hsieh, D. Qian, L. Wray, Y. Xia, Y. S. Hor, R. J. Cava, and M. Z. Hasan, Nature (London) 452, 970 (2008).
${ }^{7}$ D. Hsieh, Y. Xia, L. Wray, D. Qian, A. Pal, J. H. Dil, J. Osterwalder, F. Meier, G. Bihlmayer, C. L. Kane, Y. S. Hor, R. J. Cava, and M. Z. Hasan, Science 323, 919 (2009).

${ }^{8}$ H. Zhang, C.-X. Liu, X.-L. Qi, X. Dai, Z. Fang, and S.-C. Zhang, Nat. Phys. 5, 438 (2009).

${ }^{9}$ Y. Xia, D. Qian, D. Hsieh, L. Wray, A. Pal, H. Lin, A. Bansil, D. Grauer, Y. S. Hor, R. J. Cava, and M. Z. Hasan, Nat. Phys. 5, 398 (2009).

${ }^{10}$ Y. L. Chen, J. G. Analytis, J.-H. Chu, Z. K. Liu, S.-K. Mo, X. L. Qi, H. J. Zhang, D. H. Lu, X. Dai, Z. Fang, S. C. Zhang, I. R. Fisher, Z. Hussain, and Z.-X. Shen, Science 325, 178 (2009).

${ }^{11}$ D. Hsieh, Y. Xia, D. Qian, L. Wray, F. Meier, J. H. Dil, J. Osterwalder, L. Patthey, A. V. Fedorov, H. Lin, A. Bansil, 
D. Grauer, Y. S. Hor, R. J. Cava, and M. Z. Hasan, Phys. Rev. Lett. 103, 146401 (2009).

${ }^{12}$ H. Lin, L. A. Wray, Y. Xia, S. Jia, R. J. Cava, A. Bansil, and M. Z. Hasan, Nature Mater. 9, 546 (2010).

${ }^{13}$ Stanislav Chadov, Xiaoliang Qi, Jürgen Küler, Gerhard H. Fecher, Claudia Felser, and Shou Cheng Zhang, Nature Mater. 9, 541 (2010).

${ }^{14}$ H. Lin, R. S. Markiewicz, L. A. Wray, L. Fu, M. Z. Hasan, and A. Bansil, e-print arXiv:1003.2615 (to be published).

${ }^{15}$ Binghai Yan, Chao-Xing Liu, Hai-Jun Zhang, Chi-Yung Yam, Xiao-Liang Qi, Thomas Frauenheim, and Shou-Cheng Zhang, e-print arXiv:1003.0074 (to be published).

${ }^{16}$ L. Fu and C. L. Kane, Phys. Rev. Lett. 100, 096407 (2008).

${ }^{17}$ X.-L. Qi, T. L. Hughes, and S.-C. Zhang, Phys. Rev. B 78, 195424 (2008).

${ }^{18}$ A. M. Essin, J. E. Moore, and D. Vanderbilt, Phys. Rev. Lett. 102, 146805 (2009).

${ }^{19}$ B. Seradjeh, J. E. Moore, and M. Franz, Phys. Rev. Lett. 103, 066402 (2009).

${ }^{20}$ C. L. Kane and E. J. Mele, Phys. Rev. Lett. 95, 226801 (2005).

${ }^{21}$ C. L. Kane and E. J. Mele, Phys. Rev. Lett. 95, 146802 (2005).

${ }^{22}$ Yugui Yao, Fei Ye, Xiao-Liang Qi, Shou-Cheng Zhang, and Zhong Fang, Phys. Rev. B 75, 041401 (2007).

${ }^{23}$ Wen-Yu Shan, Hai-Zhou Lu, and Shun-Qing Shen, New J. Phys. 12, 043048 (2010).

${ }^{24}$ Shun-Qing Shen, Wen-Yu Shan, and Hai-Zhou Lu, e-print arXiv:1009.5502 (to be published).
${ }^{25}$ Pavan Hosur, Shinsei Ryu, and Ashvin Vishwanath, Phys. Rev. B 81, 045120 (2010).

${ }^{26}$ The conditions can also be simplified as $\frac{B}{M}<-\frac{1}{8}$ for the QSH insulator and $\frac{B}{M}>-\frac{1}{8}$ for the trivial insulator with $M \neq 0, B \neq 0$, and $\frac{B}{M} \neq-\frac{1}{4}$. Here, in the range of the QSH insulator, $\frac{B}{M}=-\frac{1}{4}$ is also a critical transition point, where the bands close and the system becomes a semimetal. Although the systems at the two sides are still a topological nontrivial insulator, the gapless states appear at $k_{x}=0\left(k_{x}=\pi\right)$ for $\frac{B}{M}>-\frac{1}{4}\left(\frac{B}{M}<-\frac{1}{4}\right)$.

${ }^{27}$ Ken Ichiro Imura, Ai Yamakage, Shijun Mao, Akira Hotta, and Yoshio Kuramoto, Phys. Rev. B 82, 085118 (2010).

${ }^{28}$ J. Li, R.-L. Chu, J. K. Jain, and S.-Q. Shen, Phys. Rev. Lett. 102, 136806 (2009).

${ }^{29}$ H. Jiang, L. Wang, Q.-F. Sun, and X.-C. Xie, Phys. Rev. B 80, 165316 (2009)

${ }^{30}$ C. W. Groth, M. Wimmer, A. R. Akhmerov, J. Tworzydlo, and C. W. J. Beenakker, Phys. Rev. Lett. 103, 196805 (2009).

${ }^{31}$ H.-M. Guo, G. Rosenberg, G. Refael, and M. Franz, Phys. Rev. Lett. 105, 216601 (2010).

${ }^{32}$ L. Fu and C. L. Kane, Phys. Rev. B 76, 045302 (2007).

${ }^{33}$ Zhigang Wang and Ping Zhang, New J. Phys. 12, 043055 (2010).

${ }^{34}$ D. N. Sheng, Z. Y. Weng, L. Sheng, and F. D. M. Haldane, Phys. Rev. Lett. 97, 036808 (2006).

${ }^{35}$ Roger S. K. Mong, Andrew M. Essin, and Joel E. Moore, Phys. Rev. B 81, 245209 (2010). 